\title{
Low-temperature behavior of disordered magnetic impurities: Distribution of effective Kondo temperatures
}

\author{
A.A. Zvyagin ${ }^{1,2}$ and A.V. Makarova ${ }^{3,1}$ \\ ${ }^{1}$ B. Verkin Institute for Low Temperature Physics and Engineering of the National Academy \\ of Science of Ukraine, 47 Lenin Ave., Kharkov 61103, Ukraine \\ ${ }^{2}$ Max-Planck-Institut für Physik komplexer Systeme, Dresden, Germany \\ E-mail: zvyagin@mpipks-dresden.mpg.de \\ ${ }^{3}$ Kharkov State Economic University, Kharkov, Ukraine
}

Received January 12, 2004

\begin{abstract}
Several rare-earth compounds, low-dimensional organic conductors, and spin chains exhibit low-temperature divergences of their magnetic susceptibility and specific heat (non-Fermi-liquid behavior). Such divergences are often related to disordered ensembles of magnetic impurities in those systems. In this work the distribution function of the effective characteristic of a single magnetic impurity, the Kondo temperature, is derived. We calculate how distributions of Kondo temperatures depend on the effective dimensionality of the problem and on the concentration of impurities.
\end{abstract}

PACS: 75.20.Hr, 75.10.Pq, 71.10.Hf

The Kondo effect [1] is the well-known example in which modern theoretical methods like renormalization group theory, the Bethe ansatz, bosonization, conformal field theory, etc. have demonstrated their strength [2,3]. It describes the effects of the exchange interaction between the spin of a magnetic impurity and spins of itinerant electrons. The crossover from the strong coupling to the weak coupling regime for a Kondo impurity manifests nonperturbative effects present in condensed matter theory.

During the last decade the interest in non-Fermi-liquid behavior of magnetic systems and metallic alloys has grown considerably. A large class of conducting nonmagnetic materials does not behave as usual Fermi liquids (i.e., with the finite magnetic susceptibility, finite value of the Sommerfeld low-temperature coefficient of the specific heat, and quadratic temperature behavior of the resistivity) at low temperatures. There are several possible reasons for such a behavior. One of the most known examples of the non-Fermi-liquid properties is the Kondo effect for multichannel ( $n$ is the number of channels) electron systems: For an impurity spin less than $n / 2$ a non-Fermi-liquid critical behavior results [4]. Another reason, which can cause the non-Fermi-liquid behavior, is the presence of the quantum critical point [5] (i.e., a phase transition governed not by the temperature but by some other parameter, like pressure, chemical substitution, etc.). In that case fluctuations of the order parameter interact with itinerant electrons and can cause low-temperature divergences of thermodynamic characteristics.

However, for most of nonstoichiometric conductors in which the non-Fermi-liquid behavior has been observed, see, e.g., the recent reviews [5-8], the magnetic susceptibility and low-temperature specific heat usually manifest logarithmic or weak power-law behavior with temperature, while their resistivity decreases linearly (or with some power-law exponent less than 2) with temperature, showing a large residual resistivity [9-19]. This is different from the predictions of the theory of the overscreened Kondo effect $[3,4]$. For example, Ref. 14 reported the results of measurements of the magnetic susceptibility, NMR Knight shift, and low-temperature specific heat. To explain the observed features it was necessary to assume some disorder, with a distribution of Kondo temperatures of magnetic impurities. The inhomogeneous distribution of localized magnetic moments was later confirmed [18] by muon spin rotation experiments. 
The above-mentioned properties and the alloy nature of the studied compounds suggest that the disorder (a random distribution of localized electrons or a random coupling to the conducting electron host) can play the main role in the low-temperature non-Fermi-liquid character of such systems. The idea of (unscreened) magnetic moments existing in disordered metallic systems has been formulated in [20-24]. It was proposed that near the metal-insulator transitions (or for the sufficiently alloyed systems far from the quantum critical point) disordered correlated conductors contain localized magnetic moments. The change in the interactions between the impurity sites and the host spins can be considered as a modification of the characteristic energy scale, the Kondo temperature $T_{K}$. At that scale the behavior of the magnetic impurity manifests the crossover from the strong coupling regime (for $T, H<<T_{K}, H$ being the magnetic field) to the weak coupling regime $T_{K} \ll H, T$. The impurity spin behaves asymptotically free in the weak coupling case, and it is screened by the spins of itinerant electrons in the strong coupling case. The random distribution of magnetic characteristics of impurities (i.e., their Kondo temperatures) may be connected either with the randomness of exchange couplings of itinerant electrons with the local moments [21] or with the randomness of the densities of conduction electron states [20]. Both types of randomness renormalize the single universal parameter, the Kondo temperature, which characterizes the state of a magnetic impurity. A thorough comparison of experimental results for non-Fermi-liquid behavior of disordered heavy fermion Kondo alloys has been performed with very good agreement with theoretical predictions of the model for distributed Kondo temperatures [17]. Later it was pointed out that the problem of the behavior of magnetic impurities with random distributions of their Kondo temperatures in metals can be solved exactly, with the help of the Bethe ansatz [24-26]. The role of the long-range (Ruderman-Kittel-Kasuya-Yosida) coupling between local moments was taken into account [25-28] (Griffiths phase [29] theory), exhibiting properties qualitatively similar to those of models with noninteracting local moments. Also, the presence of a spin-orbit interaction in some disordered heavy fermion alloys demands the study of a magnetic anisotropy, which can play an essential role in physics of disordered spin interactions [26-28,30]. Finally, it was exactly proved that for correlated electrons systems with magnetic impurities with random distributions of their couplings to the host it is also possible to introduce a distribution of effective Kondo temperatures [31] which governs the low-temperature nonFermi-liquid behavior of the system.
It was pointed out $[25,26,28,31]$ that distributions of effective Kondo temperatures for each magnetic impurity can cause divergences of the magnetic susceptibility and the Sommerfeld coefficient of the specific heat for quasi-one-dimensional organic conductors and quantum spin chains, where such behavior was observed [32-35]. To explain power-law divergences of the magnetic susceptibilities and Sommerfeld coefficients of rare-earth and actinide compounds, as well as quasi-one-dimensional organic conductors and quantum spin chains, it was necessary to use a distribution of Kondo temperatures (the strong-disorder distribution, for which the tails are rather large) which starts with the term $P\left(T_{K}\right) \propto G^{-\lambda}\left(T_{K}\right)^{\lambda-1}(\lambda<1)$ valid till some energy scale $G$ for the lowest values of $T_{K}$ [25-27,30,31]. The goal of this work is to obtain the distribution of Kondo temperatures for a system with magnetic impurities and to show how such a distribution will depend on the effective spatial dimensionality of the system (it turns out that the exponent $\lambda$ is different for three-dimensional non-Fermi-liquid heavy fermion systems and for quasi-one-dimensional organic conductors and quantum spin systems [16,32, $33,35])$.

Let us consider the model of electrons (itinerant and localized, where localization centers, i.e., $3 d, 4 f$, or $5 f$ orbitals, are distributed randomly on a hypercubic lattice with a random nearest-neighbor hopping) with the Hamiltonian [20]

$$
\mathcal{H}=\sum_{j, j^{\prime}, \sigma} t\left(j, j^{\prime}\right) c_{j, \sigma}^{\dagger} c_{j^{\prime}, \sigma}+\mathcal{H}_{\mathrm{int}},
$$

where $c_{j, \sigma}^{\dagger}\left(c_{j, \sigma}\right)$ creates (annihilates) an electron with spin $\sigma$ at site $j$, and $t\left(j, j^{\prime}\right)$ are hopping elements. The interaction part of the Hamiltonian, $\mathcal{H}_{\text {int }}=(U / 2) \sum_{j} n_{j, \sigma} n_{j,-\sigma}$, is determined by the Coulomb interaction of localized electrons (the sum is over random positions of localized electrons, and $\left.n_{j, \sigma}=c_{j, \sigma}^{\dagger} c_{j, \sigma}\right)$. Generally speaking, one can add to the Hamiltonian (1) disordered local potentials $\sum_{j, \sigma} \varepsilon_{j} n_{j \sigma}$. The hopping integrals can be approximated [36] as overlap integrals

$$
t\left(j, j^{\prime}\right)=E_{0} \exp \left(\frac{-r_{j, j^{\prime}}}{a}\right)\left(1+\frac{r_{j, j^{\prime}}}{a}+\ldots\right),
$$

where $a$ is the Bohr radius and $E_{0} \sim U$ is the effective binding energy of the dopant (localized electron). We suppose that hopping integrals are random because of the disorder of the distribution of localized electrons.

Let us consider the situation for which localized electrons are in the magnetic state, i.e., their valence is close to 1 , which is satisfied if $t^{2}\left(j, j^{\prime}\right) \rho\left(E_{F}\right)<<$ $<-\varepsilon_{j}, U+\varepsilon_{j}$ (with negative $\varepsilon_{j}$, where $\rho\left(E_{F}\right)$ is 
the density of states at the Fermi level) [2]. It is natural to assume that the density of the localized magnetic moments $n_{l}$ depends on the density of dopants $n$ as

$$
n_{l}=n \exp \left(-n / n_{\max }\right) \text {, }
$$

where $n_{\max }$ is related to the critical distance between localization centers $R_{c}$ via $n_{\max }=1 / V_{c}$. Here $V_{c}$ depends on the effective dimensionality of the distribution of localized magnetic moments. It is equal to $V_{c}=4 \pi R_{c}^{2} / 3$ for the three-dimensional case, $V_{c}=\pi R_{c}^{2}$ for the effectively two-dimensional situation, and for effectively one-dimensional distribution of localized magnetic moments one has $V_{c}=R_{c}$. Such a distribution is well-known in the theory of disordered systems (it follows from simple combinatorics), e.g., in the theory of dislocations with defects it is known as the Koehler formula [36], see also [37,38]. For large $\mathrm{n}, n_{l}$ is a decreasing function of $n$, as it must be. Using such an assumption we can find the probability $P(r)$ to find the nearest-neighbor localized moment of a given site at a distance $r$, see below. (On the other hand, $P(r)$ can be derived independently
[39], and we can obtain Eq. (3).) Obviously, the density of the localized magnetic moments can be written as

$$
n_{l}=\int_{R_{c}}^{\infty} P(r) d r
$$

Using the definition of $R_{c}$ we obtain

$$
P(r)=n \begin{cases}\pi r^{2} \exp \left(-4 \pi n r^{3} / 3\right), & d=3 \\ 2 \pi r \exp \left(-\pi n r^{2}\right), & d=2 \\ \exp (-n r), & d=1\end{cases}
$$

where $d$ is the effective dimensionality of the distribution of localized electrons, cf. [36,39]. One can check that $n_{l}$ has a maximum at $n_{\max }$, which, actually, justifies Eq. (3).

The next step is to obtain the distribution of hopping integrals $t\left(j, j^{\prime}\right)$ between localized moments (in what follows we shall denote them simply as $t$ ). Equation (2) implies $r(t) \approx a \ln \left(2 E_{0} / t\right)$. Then, using $\tilde{P}(t)=P(r)(d r / d t)$ and Eq. (5) we get

$$
\tilde{P}(t)=-\frac{a n}{t} \begin{cases}\pi a^{2} \ln ^{2}\left(E_{0} / t\right) \exp \left[-\left(4 \pi n a^{3} / 3\right) \ln ^{3}\left(E_{0} / t\right)\right], & d=3 \\ 2 \pi a \ln \left(E_{0} / t\right) \exp \left[-\pi n a^{2} \ln ^{2}\left(E_{0} / t\right)\right], & d=2 \\ \exp \left[-n a \ln \left(E_{0} / t\right)\right], & d=1\end{cases}
$$

for the three-, two-, and one-dimensional cases, respectively.

The Kondo temperature of the localized magnetic moment can be written as [2]

$$
T_{K}=\sqrt{\frac{2 U \rho\left(E_{F}\right)}{\pi}} t \exp \left(-\frac{|\varepsilon|(\varepsilon+U)}{2 U \rho\left(E_{F}\right) t^{2}}\right) \approx D \exp \left(-\frac{|\varepsilon|(\varepsilon+U)}{2 U \rho\left(E_{F}\right) t^{2}}\right),
$$

where we have assumed homogeneous distributions of the local potentials $\varepsilon_{j}=\varepsilon$ and introduced the low-energy cutoff $D$, as usual for the Kondo problem [2]. Notice that for $\varepsilon=0$ one can define the Kondo temperature as $T_{K}=D \exp \left(-2 U / \rho\left(E_{F}\right) t^{2}\right)$. Then Eq. (7) implies

$$
\frac{d T_{K}}{d t} \approx-\frac{t}{2 T_{K} \ln \left(T_{K} / D\right)} .
$$

Defining $x=\ln \left(D / T_{K}\right)$ and $A=8 U \rho\left(E_{F}\right) E_{0}^{2} /|\varepsilon|(\varepsilon+U)$, we obtain the distributions of Kondo temperatures

$$
\bar{P}\left(T_{K}\right)=\frac{Z_{d} n}{2 n_{\max } T_{K} x} \begin{cases}(3 / 16) \ln ^{2}(A x) \exp \left[-\left(n / 8 n_{\max }\right) \ln ^{3}(A x)\right], & d=3 \\ \ln (A x) \exp \left[-\left(n / 4 n_{\max }\right) \ln ^{2}(A x)\right], & d=2 \\ \exp \left[-\left(n / 2 n_{\max }\right) \ln (A x)\right], & d=1\end{cases}
$$

for the three-, two-, and one-dimensional cases, respectively, where $Z_{d}$ are normalization constants. Observe that the divergence of $\bar{P}\left(T_{K}\right)$ as $T_{K} \rightarrow 0$ (due to the factor $T_{K}^{-1}$ ) is weakened by logarithmic factors like $\ln \left(T_{K} / D\right)$. This is why $\bar{P}\left(T_{K}\right)$ can be normalized to unity over some interval $0 \leq T_{K} \leq T_{\max }$, where $T_{\max }$ is given by $T_{\max }=D \exp \left(-|\varepsilon|(\varepsilon+U) / 8 U \rho\left(E_{F}\right) E_{0}^{2}\right)$. $T_{\max }$ can be related to the parameter $G$, see above. Obviously the distributions of Kondo temperatures depend on the parameters of impurity $\varepsilon$, the constant 
of Coulomb repulsion $U$, the density of states of itinerant electrons at the Fermi level, the Bohr radius, and the density of localized orbitals. However, Eqs. (9) imply that such a set of parameters is realized in two main governing parameters: $n / n_{\max }$ and $A$.

It turns out that for large enough intervals of values of $T_{K}$ the distribution $\bar{P}\left(T_{K}\right)$ is proportional to $T_{K}^{\lambda-1}$, i.e., it is of the form used in [25-27,30,31] without derivation, based on experimental results. The exponent $\lambda<1$ is determined by $A$ and $n / n_{\max }$. The plots of the logarithms of $\bar{P}\left(T_{K}\right)$ as functions of $x$ are presented in Fig. 1, $a$ for one-, two-, and three-dimensional situations (we did not take into account the terms in Eqs. (9) which do not depend on $x$ here; those terms yield only constant shifts). Here we used the parameters $D, \varepsilon, U, E_{0}$, appropriate for real disordered rare-earth and actinide systems, which produces $A \approx 1$. Also, according to experiments, one can expect $n / n_{\max } \approx 0.01$. Notice that the maximal values of intervals of $T_{K}, T_{\max }$, depend on $A$ via $T_{\max }=$ $=D \exp (-1 / A)$, and, hence, $x_{\min }=1 / A$. This is why we present here the results for large enough values of $x$ (i.e., for small $T_{K}$ ), that the distributions in the considered intervals are reminiscent of the power laws $\propto T_{K}^{1-\lambda}$. One can see that the exponents of the distributions depend on the effective dimensionality of the distribution of the localized electrons.

To illustrate how the effective exponents depend on $A$ and on the concentration of localized electrons $n$, we present results for the logarithms of the distributions of Kondo temperatures for $A=5, n / n_{\max }=0.01$ in Fig. $1, b$ and for $A=1, n / n_{\max }=0.5$ in Fig. 1,c. The results show that the effective exponents $\lambda$ are large ( $\lambda \propto 0.8$ for the realistic case $A \sim 1, n / n_{\max } \sim 0.01$ for three space dimensions). It is too large compared to the experiments on three-dimensional heavy fermion systems [14,16-18], for which values of $\lambda=0.6-0.85$ were observed. Also, for some values of the parameters a value $\lambda>1$ was obtained, which implies the absence of low-temperature divergences in such cases. The reason for such an overestimation can lie in the disorder in the distribution of local potentials of localizated electrons, $\varepsilon_{j}$, which was not taken into account in our description. However, smaller values of $\lambda(0.26-0.42)$ have been observed for low-dimensional organic conductors and spin chains [32,33]. It turns out that large values of $\lambda \sim 0.2$ were observed for disordered ensembles of impurities close to a metal-insulator transition.

Such a distribution of Kondo temperatures produces non-Fermi-liquid behavior of many characteristics of the system [25-27,31]. For example, the ground state magnetization of the system behaves as $M \propto(H / G)^{\lambda}$ ( $H$ is an external magnetic field $)$, i.e., essentially in a nonlinear way. The static magnetic susceptibility at low temperatures is divergent as $T^{\lambda-1} / G^{\lambda}$. Also, the nonlinear behavior of the electron specific heat $c \sim(T / G)^{\lambda}$ implies divergent behavior of the Sommerfeld coefficient. The low-temperature behavior of the correlation length is proportional to $(G / T)^{\lambda}$, which implies non-Fermi-liquid behavior of the low-temperature resistivity. Finally, the imaginary part of the dynamic magnetic susceptibility is also divergent as $G^{\lambda-2} T^{1-\lambda} g(\omega / T)$, where $g(x)=$ $=x \int_{1}^{\infty} d y / y^{\lambda-1}\left(x^{2}+y^{2}\right)$ is a universal scaling function [25].

Summarizing, in this work we have calculated the distribution of effective Kondo temperatures for a
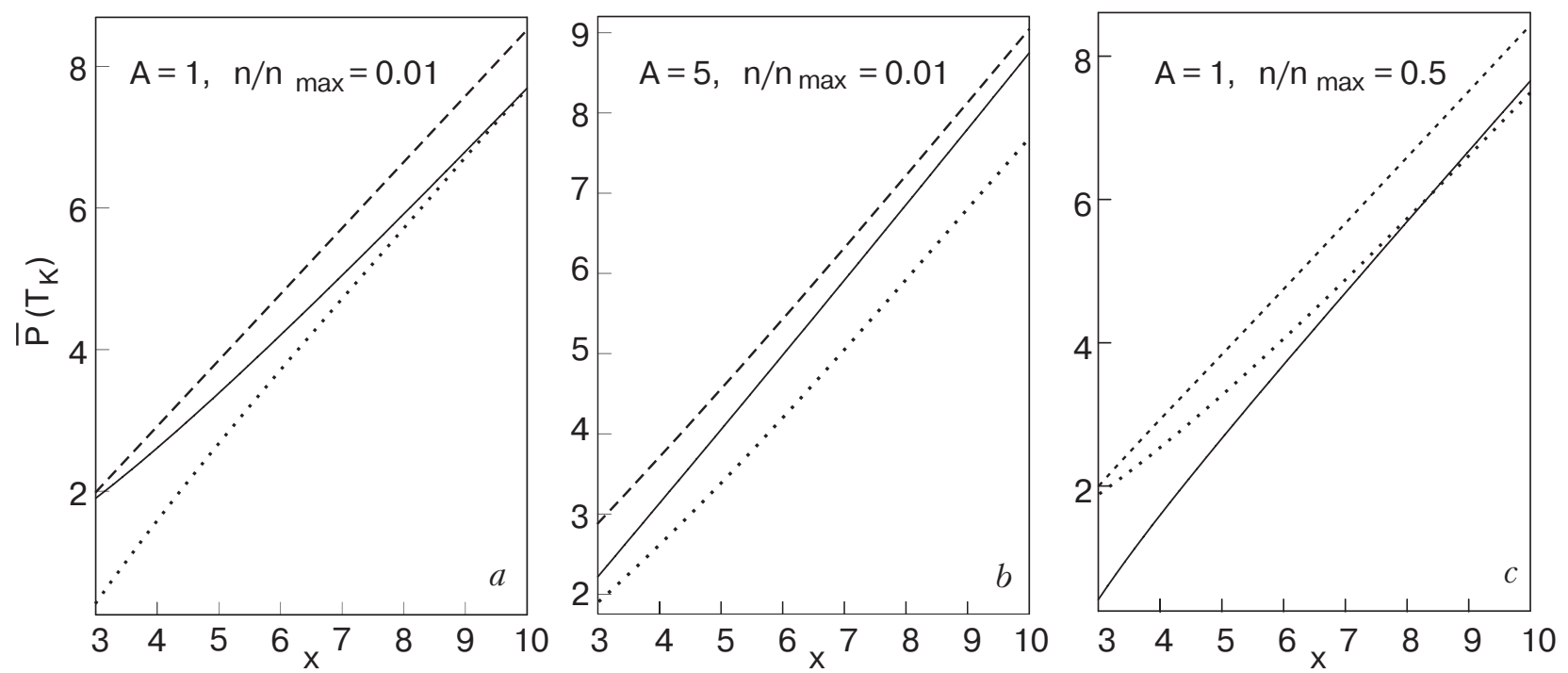

Fig. 1. Logarithms of the distributions of the Kondo temperatures for the three-dimensional (solid line), two-dimensional (dotted line), and one-dimensional (dashed line) cases. 
system of correlated electrons in which itinerant electrons are hybridized with randomly distributed localized electrons ( $3 d, 4 f$, or $5 f$ orbitals). Those distributions depend on many parameters: concentration of localized orbitals, their Bohr radii, Coulomb interaction of localized electrons on orbitals, effective energies of localized electrons, bandwidths of itinerant electrons, etc. However, we have shown that only two parameters effectively govern the behavior of the distribution functions of Kondo temperatures. One of those parameters is the ratio of the density of localized electrons to the maximal density (in a hypercubic lattice). The other parameter is related to the maximal Kondo temperatures for which such an approach can be applied. We have shown that distributions of Kondo temperatures depend on the effective dimensionality of the disordered localized electrons. Due to such a disordered behavior of magnetic impurities many thermodynamic and kinetic characteristics of the considered systems manifest non-Fermi-liquid features in their low-energy characteristics.

1. J. Kondo, in: Solid State Physics: Advances in Research and Applications, F. Seitz, D. Turnbull, and H. Ehrenreich (eds.), Academic, NY (1969), V. 23, P. 184.

2. A.M. Tsvelick and P.B. Wiegmann, Adv. Phys. 32, 453 (1983).

3. I. Affleck, Acta Phys. Polon. 26, 1869 (1995).

4. P. Schlottmann and P.D. Sacramento, Adv. Phys. 42, 641 (1993).

5. F. Steglich, J. Magn. Magn. Matter. 226, 1 (2001).

6. H. v. Löhneysen, F. Huster, S. Mock, A. Neubert, T. Pietrus, M. Sieck, O. Stockert, and M.M. Waffenschmidt, Physica B230-232, 550 (1997).

7. M.B. Maple, M.C. de Andrade, J. Herrmann, Y. Dalichaouch, D.A. Gajewski, C.L. Seaman, R. Chau, R. Movshovich, M.C. Aronson, and R. Osborn, J. Low Temp. Phys. 99, 223 (1995).

8. G.R. Stewart, Rev. Mod. Phys. 73, 797 (2001).

9. C. Seaman, M.B. Maple, B.W. Lee, S. Ghamaty, M.S. Torikachvili, J.-S. Kang, L.Z. Liu, J.W. Allen, and D.L. Cox, Phys. Rev. Lett. 67, 2882 (1991).

10. G.R. Stewart, Phys. Rev. B47, 3208 (1993).

11. B. Andraka, Phys. Rev. B49, 348 (1994).

12. B. Andraka, Phys. Rev. B49, 3589 (1994).

13. M.C. Aronson, R. Osborn, R.A. Robinson, J.W. Lynn, R. Chau, S.L. Seaman, and M.B. Maple, Phys. Rev. Lett. 75, 725 (1995).

14. O.O. Bernal, D.E. MacLaughlin, H.G. Lukefahr, and B. Andraka, Phys. Rev. Lett. 75, 2023 (1995).

15. K. Matsuhira, T. Sakakibara, and H. Amitsuka, Physica B206-207, 326 (1995).
16. M.C. de Andrade, R. Chau, R.P. Dickey, N.R. Dilley, E.J. Freeman, D.A. Gajewski, M.B. Maple, R. Movshovich, A.H. Castro Neto, G.E. Castilla, and B.A. Jones, Phys. Rev. Lett. 81, 5620 (1998).

17. C.H. Booth, D.E. MacLaughlin, R.H. Heffner, R. Chau, M.B. Maple, and G.H. Kwei, Phys. Rev. Lett. 81, 3960 (1998).

18. D.E. MacLaughlin, R.H. Heffener, G.J. Nieuwenhuys, G.M. Luke, Y. Fudamoto, Y.J. Uemura, R. Chau, M.B. Maple, and B. Andraka, Phys. Rev. B58, R11849 (1998).

19. L. Shlyk, J.C. Waerenborgh, P. Estrela, L.E. De Long, A. de Visser, and M. Almeida, J. Phys.: Condensed Matter 11, 3525 (1999).

20. R.N. Bhatt and D.S. Fisher, Phys. Rev. Lett. 68, 3072 (1992).

21. V. Dobrosavljević, T.R. Kirkpatrick, and G. Kotliar, Phys. Rev. Lett. 69, 1113 (1992).

22. E. Miranda, V. Dobrosavljević, and G. Kotliar, Phys. Rev. Lett. 78, 290 (1997).

23. E. Miranda, V. Dobrosavljević, and G. Kotliar, J. Phys.: Condensed Matter 8, 9871 (1996).

24. A. Klümper and A.A. Zvyagin, Phys. Rev. Lett. 81, 4975 (1998).

25. A.A. Zvyagin, Phys. Rev. B62, R6069 (2000).

26. A.A. Zvyagin, Fiz. Nizk. Temp. 28, 1274 (2002) [Low Temp. Phys. 28, 907 (2002)].

27. A.H. Castro Neto, G. Castilla, and B.A. Jones, Phys. Rev. Lett. 81, 3531 (1998).

28. A. Klümper and A.A. Zvyagin, J. Phys.: Condensed Matter 12, 8705 (2000).

29. R.B. Griffiths, Phys. Rev. Lett. 23, 17 (1969).

30. A.H. Castro Neto and B.A. Jones, Phys. Rev. B62, 14975 (2000).

31. A.A. Zvyagin, Phys. Rev. B63, 033101 (2001).

32. L.N. Bulaevsky, A.V. Zvarykina, Yu. S. Karimov, R.B. Lyubosky, and I.F. Shchegolev, Zh. Eksp. Teor. Fiz. 62, 725 (1972) [JETP 35, 384 (1972)].

33. K. Ikegami, S. Kuroda, M. Saito, K. Saito, M. Sugi, T. Nakamura, M. Matsumoto, and Y. Kawabata, Phys. Rev. B35, 3667 (1987).

34. K. Mukai, K. Suzuki, K. Ohara, J.B. Jamali, and N. Achiwa, J. Phys. Soc. Jpn. 68, 3078 (1999).

35. S.V. Demishev, R.V. Bunting, L.I. Leonyuk, E.D. Obraztsova, A.A. Pronin, N.E. Sluchanko, N.A. Samarin, and S.V. Terekhov, Pis'ma v Zh. Eksp. Teor. Fiz. 73, 36 (2001) [JETP Lett. 73, 31 (2001)].

36. J.S. Koehler, in: Imperfections in Nearly Perfect Crystals, New York (1952), P. 197.

37. C.L. Bauer, Philos. Mag. 11, 827 (1965).

38. A. Granato, and K. Lücke, J. Appl. Phys. 27, 583 (1956).

39. M. Lakner, H. v. Löhneysen, A. Langenfeld, and P. Wölfle, Phys. Rev. B50, 17064 (1994). 